\title{
A Methodology for Transient State Estimation based on Numerical Derivatives, Optimal Monitoring and Filtered Measurements
}

\author{
I. Molina-Moreno, Member, IEEE, A. Medina, Senior Member, IEEE, R. Cisneros-Magaña, Member, \\ IEEE, and O. Anaya-Lara, Member, IEEE
}

\begin{abstract}
This paper proposes a methodology for transient state estimation in power systems. The proposed methodology is formulated using approximation methods for derivatives to relate the state variables to measurements. It does not require knowledge of the steady state to establish the pre-disturbance operation conditions. The method uses an optimal monitoring system based on topological analysis to obtain full observability. A saving index is introduced to analyze the effectiveness of the instrumentation used. The adverse effect of noisy measurements in the estimation process is mitigated using an Infinite Impulse Response (IIR) filter. A transient index is introduced to estimate the fault location. The transient state estimation is assessed using two test systems. The results are validated through direct comparison against those obtained by simulation using SimPowerSystems toolbox of Simulink®. With the proposed methodology, the transient state estimation can be obtained with an important saving in the implementation of the measuring system and with considerably less computational effort.
\end{abstract}

Index Terms-Approximation methods, infinite impulse response filters, observability, optimal monitoring, steady state, transient state estimation.

\section{INTRODUCTION}

$\mathrm{P}^{\mathrm{o}}$ OWER quality is a matter of concern to utilities, end users and equipment suppliers [1]. Power quality state estimation (PQSE) deals with a limited number of measuring devices [2-3]. Issues that encompass PQSE are fundamental frequency state estimation [4-5], harmonic state estimation [6-7], voltage sag state estimation [8-9], and transient state estimation (TSE) [10-15]. This research work, deals with TSE in power systems.

Electromagnetic transients or simply transients are severe short-time variations of voltage and current in a power system. They are mainly generated by disturbance events such as line faults, sudden load or switching events [1]. The resulting

I. Molina-Moreno is with the Facultad de Ingeniería Eléctrica, División de Estudios de Posgrado, Universidad Michoacana de San Nicolás de Hidalgo, Morelia, México (e-mail: imolina@dep.fie.umich.mx).

A. Medina is with the Facultad de Ingeniería Eléctrica, División de Estudios de Posgrado, Universidad Michoacana de San Nicolás de Hidalgo, Morelia, México (e-mail: amedinr@gmail.com).

R. Cisneros-Magaña is with the Facultad de Ingeniería Eléctrica, División de Estudios de Posgrado, Universidad Michoacana de San Nicolás de Hidalgo, Morelia, México (e-mail: rcisneros@dep.fie.umich.mx).

O. Anaya-Lara is with the Institute for Energy and Environment, Department of Electronic and Electrical Engineering, University of Strathclyde, Glasgow, UK (e-mail: olimpo.anaya-lara@strath.ac.uk). energy exchanges, subject the network components to higher stresses, lead to excessive current or voltage variations which can be damaging [16].

Computational programs such as Electromagnetic Transients with Direct Current (EMTDC), Electromagnetic Transients Program (EMTP), Alternative Transients Program (ATP), or similar have been used to simulate the behavior of the network during a specific disturbance event. Thus, the adverse effects of transients in power systems can be adequately predicted [17-19]. Whereas the transient simulation is used to analyze the consequences of a disturbance in a power system, TSE is used to identify the cause of transients in the power system [10-15].

Reference [10] introduces the concept of TSE as the inverse transient simulation problem, i.e. the main objective is to identify the disturbance that caused transients in the power system. The method describes the power system by a firstorder ordinary differential equation (ODE) set where the busbar voltages and inductor currents are the state variables. The model order of the system is high since the power system is usually large. The ODE set is discretized using backward Euler integration formula; thus, an initial state is needed. The solution is obtained after considerable computational effort. In addition, more computational effort to reach the steady state, necessary to determine the pre-disturbance time interval, is required.

Reference [11] formulates the TSE problem as in [10], i.e. the node voltages and branch currents are selected as state variables. The initial state is also zero; however, the periodic steady state is rapidly reached as the steady state is obtained through a Newton method based on a Numerical Differentiation process, Poincaré map, and extrapolation to the limit cycle [20]. The method uses singular value decomposition (SVD) to solve the TSE. The SVD is used when $m<n$ where $m$ is the number of measurements and $n$ the number of state variables [21-22]. However, the computational effort is increased as the computational requirement for SVD is of the order $m n^{2}$.

References [12-14] present the problem formulation for the TSE where only the node voltages are taken as state variables. The formulation is based on the numerical integrator substitution method which requires of an initial state. The initial state is set to zero; hence, the algorithms may need considerable computational effort to reach the steady ready. 
They also use the SVD to solve the TSE.

Reference [15] proposes a method based on Kalman filter to assess the TSE in power systems. The method exploits the half-wave symmetry in the voltage and current waveforms to make more efficient the numerical differentiation method to obtain the periodic steady state solution of a power network, which is the base to generate the pre-disturbance time interval.

In this contribution, an alternative methodology for TSE assessment in power systems is proposed. The problem TSE is formulated using approximation methods for derivatives instead of integration ones. The method includes an optimal monitoring system based on topological analysis to obtain full observability. The noise in measurements is mitigated using an IIR filter. Computational effort to obtain the pre-disturbance steady state is not required.

The rest of the paper is organized as follows: Section 2 details the proposed TSE methodology; Section 3 describes the test systems used to conduct the case studies; Section 4 analyzes the obtained results, which are in turn validated and Section 5 draws the main conclusions from this research work.

\section{Proposed TSE Methodology}

The main stages for the proposed TSE methodology are as follows:

- Mathematical formulation for the TSE problem.

- Optimal monitoring.

- Filtering of noisy measurements during TSE

- Algorithm for TSE.

\section{A. Mathematical formulation to TSE problem}

The proposed mathematical formulation to TSE problem is given as follows,

$$
\mathbf{x}=\mathbf{H}^{-1} \mathbf{z}+\mathbf{e}
$$

where $\mathbf{x} \in \mathbb{R}^{n}$ is the state vector that contains the node voltages to be estimated; $\mathbf{z} \in \mathbb{R}^{m}$ is the measurement vector; $\mathbf{H} \in \mathbb{R}^{m \times n}$ is the measuring matrix that relates the state variables to measurements; and $\mathbf{e} \in \mathbb{R}^{m}$ is the error vector that represents the error associated to the measuring process, generally assumed to be zero mean white Gaussian noise. The idea is to determine $\mathbf{x}$ as a function of measurement quantities. $\mathbf{H}$ will not be inverted, instead, based on parameters of the power system, $\mathbf{H}^{-1}$ is directly developed.

The power system is modeled by an ODE set. However, it can be transformed to difference equations if time $t$ is defined as,

$$
t[k]=k T_{\mathrm{S}}=t^{k}
$$

where $T_{\mathrm{S}}$ is the sampling period, and $k$ is the $k$-th sample number. The notation of $k T_{\mathrm{S}}$ will be represented only by the super index $k$. In addition, numerical derivatives are needed to transform the ODE set into difference equations. There are several formulas suitable to obtain the derivative of a function [23]. The basic formulas are known as forward-difference formula if $T_{\mathrm{S}}>0$ and backward-difference formula if $T_{\mathrm{S}}<$ 0 . These formulas generate an error $O\left(T_{\mathrm{S}}\right)$. In this research, the three-point midpoint formula is used since it generates an error $O\left(T_{\mathrm{S}}^{2}\right)$. Hence, the derivative of the function $g$ at $t=k T$ is,

$$
g^{\prime}[k]=\left(g^{k+1}-g^{k-1}\right) / 2 T_{\mathrm{S}}+O\left(T_{\mathrm{S}}^{2}\right) .
$$

The second derivative of the function $g$ at $t=k T$ can be obtained using the second derivative midpoint formula. This formula also generates an error $O\left(T_{\mathrm{S}}^{2}\right)$ and is defined as,

$$
g^{\prime \prime}[k]=\left(g^{k+1}-2 g^{k}+g^{k-1}\right) / T_{\mathrm{S}}^{2}+O\left(T_{\mathrm{S}}^{2}\right) .
$$

The relevant system components are modeled in their equivalents:

1) Two busbars connected through a transmission line: The transmission line is usually modeled through distributed parameters but it can be modeled as an equivalent $\pi$-circuit, as there is only interest in the behavior at the input and at the output ends of the line. The modeling is extended to threephase networks using matrix notation for the representation of parameters, as shown in Fig. 1(a). Here, $\mathbf{R}$ contains the self and mutual resistances, $\mathbf{L}$ the self and mutual inductances, $\mathbf{C}$ the self and mutual capacitances, and $\mathbf{G}$ the self and mutual admitances, necessary for the equivalent $\pi$-circuit [24]. In the parameters $\mathbf{R}, \mathbf{L}, \mathbf{C}$ and $\mathbf{G} \in \mathbb{R}^{3 \times 3}$, the diagonal values correspond to self values and the off-diagonal values to mutual values.

The series current $\mathbf{i} \in \mathbb{R}^{3 \times 3}$, and the shunt currents, $\mathbf{i}_{G}$ and $\mathbf{i}_{C} \in \mathbb{R}^{3 \times 3}$ are not possible measurements since they are physically not available. However, by applying circuit theory in the continuous-time, the busbar voltages, $\mathbf{v}_{s}$ and $\mathbf{v}_{r} \in \mathbb{R}^{3 \times 3}$, can be related as,

$$
\begin{gathered}
\mathbf{v}_{s}-\mathbf{v}_{r}=\mathbf{R i}+\mathbf{L i}^{\prime}, \\
\text { where } \mathbf{v}_{s}=\left[\begin{array}{l}
v_{s_{1}} \\
v_{s_{2}} \\
v_{s_{3}}
\end{array}\right], \quad \mathbf{v}_{r}=\left[\begin{array}{l}
v_{r_{1}} \\
v_{r_{2}} \\
v_{r_{3}}
\end{array}\right], \quad \mathbf{R}=\left[\begin{array}{lll}
R_{11} & R_{12} & R_{13} \\
R_{21} & R_{22} & R_{23} \\
R_{31} & R_{32} & R_{33}
\end{array}\right], \\
\mathbf{i}=\left[\begin{array}{l}
i_{1} \\
i_{2} \\
i_{3}
\end{array}\right], \mathbf{L}=\left[\begin{array}{lll}
L_{11} & L_{12} & L_{13} \\
L_{21} & L_{22} & L_{23} \\
L_{31} & L_{32} & L_{33}
\end{array}\right], \text { and } \mathbf{i}^{\prime}=\left[\begin{array}{l}
i_{1}^{\prime} \\
i_{2}^{\prime} \\
i_{3}^{\prime}
\end{array}\right] .
\end{gathered}
$$

By applying the Kirchhoff current law (KCL) at busbar $s$, the vector of sending end currents $\mathbf{i}_{s}$ is defined as,

$$
\mathbf{i}_{s}=\mathbf{i}+\mathbf{i}_{\mathrm{G} s}+\mathbf{i}_{\mathrm{C} s},
$$

where $\mathbf{i}_{s}=\left[\begin{array}{l}i_{s_{1}} \\ i_{s_{2}} \\ i_{s_{3}}\end{array}\right], \mathbf{i}_{\mathrm{G} s}=\left[\begin{array}{l}i_{\mathrm{G} s_{1}} \\ i_{\mathrm{G} s_{2}} \\ i_{\mathrm{G} s_{3}}\end{array}\right]$, and $\mathbf{i}_{\mathrm{C} s}=\left[\begin{array}{l}i_{\mathrm{C} s_{1}} \\ i_{\mathrm{C} s_{2}} \\ i_{\mathrm{C} s_{3}}\end{array}\right]$.

The continuous-time current in the capacitor $\mathbf{C}$, connected at busbar $s$, is given by,

$$
\mathbf{i}_{\mathrm{Cs}}=\mathbf{C} \mathbf{v}_{S}^{\prime}
$$

where $\mathbf{C}=\left[\begin{array}{lll}C_{11} & C_{12} & C_{13} \\ C_{21} & C_{22} & C_{23} \\ C_{31} & C_{32} & C_{33}\end{array}\right]$, and $\mathbf{v}_{s}^{\prime}=\left[\begin{array}{c}v_{s_{1}}^{\prime} \\ v_{s_{2}}^{\prime} \\ v_{s_{3}}^{\prime}\end{array}\right]$.

By applying the Ohm's law, the continuous-time current in an admittance $\mathbf{G}$, connected at busbar $s$ is given by,

$$
\mathbf{i}_{\mathrm{Gs}}=\mathbf{G v}_{s}
$$

where $\mathbf{G}=\left[\begin{array}{lll}G_{11} & G_{12} & G_{13} \\ G_{21} & G_{22} & G_{23} \\ G_{31} & G_{32} & G_{33}\end{array}\right]$.

By substituting (7) and (8) in (6) and solving for i, gives, 


$$
\mathbf{i}=\mathbf{i}_{\mathbf{s}}-\mathbf{G v}_{\mathbf{s}}-\mathbf{C} \mathbf{v}_{\mathbf{s}}^{\prime} .
$$

The derivate of (9) is

$$
\mathbf{i}^{\prime}=\mathbf{i}_{s}^{\prime}-\mathbf{G v}_{s}^{\prime}-\mathbf{C} \mathbf{v}_{s}^{\prime \prime} .
$$

By substituting (9) and (10) in (5) and solving for $\mathbf{v}_{r}$ gives, $\mathbf{v}_{r}=(\mathbf{I}+\mathbf{R G}) \mathbf{v}_{s}+(\mathbf{R C}+\mathbf{L G}) \mathbf{v}_{s}^{\prime}+\mathbf{L} \mathbf{C} \mathbf{v}_{s}^{\prime \prime}-\mathbf{R i}_{s}-\mathbf{L} \mathbf{i}_{s}^{\prime}$

If $\mathbf{v}_{s}$ and $\mathbf{i}_{s}$ are measured, $\mathbf{v}_{r}$ can be estimated. Let $\mathbf{z}_{\mathbf{v}_{S}}$ the busbar voltage and $\mathbf{z}_{\mathbf{i}_{s}}$ the sending end current measurements. Equation (11) is now left as a function of measurements, i.e.

$\mathbf{v}_{r}=(\mathbf{I}+\mathbf{R G}) \mathbf{z}_{\mathbf{v}_{S}}+(\mathbf{R C}+\mathbf{L G}) \mathbf{z}_{\mathbf{v}_{S}}^{\prime}+\mathbf{L} \mathbf{C} \mathbf{z}_{\mathbf{v}_{S}}^{\prime \prime}-\mathbf{R z}_{\mathbf{i}_{s}}-\mathbf{L} \mathbf{z}_{\mathbf{i}_{S}}^{\prime}(12)$

By applying (3) and (4) to discretize (12) results in, $\mathbf{v}_{r}^{k}=(\mathbf{I}+\mathbf{R G}) \mathbf{z}_{\mathbf{v}_{S}}^{k}+\frac{(\mathbf{R C}+\mathbf{L G})\left(\mathbf{z}_{\mathbf{v}_{S}}^{k+1}-\mathbf{z}_{\mathbf{V}_{S}}^{k-1}\right)}{2 T_{S}}+\frac{\mathbf{L C}\left(\mathbf{z}_{\mathbf{v}_{S}}^{k+1}-2 \mathbf{z}_{\mathbf{v}_{S}}^{k}+\mathbf{z}_{\mathbf{V}_{S}}^{k-1}\right)}{T_{\mathbf{S}}^{2}}-$ $\mathbf{R} \mathbf{z}_{\mathbf{i}_{S}}^{k}-\frac{\mathbf{L}\left(\mathbf{z}_{\mathbf{i}_{S}}^{k+1}-\mathbf{z}_{\mathbf{i}_{S}}^{k-1}\right)}{2 T_{S}}$

Reordering (13) gives,

$$
\mathbf{v}_{r}^{k}=\mathbf{c}_{1, s-r} \mathbf{z}_{\mathbf{v}_{S}}^{k}+\mathbf{c}_{2, s-r} \mathbf{z}_{\mathbf{v}_{S}}^{k+1}+\mathbf{c}_{3, s-r} \mathbf{z}_{\mathbf{v}_{S}}^{k-1}+\mathbf{b}_{1, \mathrm{~s}} \mathbf{z}_{\mathbf{i}_{s}}^{k}+
$$$$
\mathbf{b}_{2, s} \mathbf{z}_{\mathbf{i}_{S}}^{k+1}+\mathbf{b}_{3, s} \mathbf{z}_{\mathbf{i}_{S}}^{k-1}
$$

where $\mathbf{c}_{1, s-r}=\mathbf{I}+\mathbf{R G}-2 \mathbf{L C} / T_{\mathrm{S}}^{2}$,

$\mathbf{c}_{2, s-r}=(\mathbf{R C}+\mathbf{L G}) / 2 T_{\mathrm{S}}+\mathbf{L C} / T_{\mathrm{S}}^{2}$,

$\mathbf{c}_{3, S-r}=\mathbf{L C} / T_{\mathrm{S}}^{2}+(\mathbf{R C}+\mathbf{L G}) / 2 T_{\mathrm{S}}, \mathbf{b}_{1, \mathrm{~s}}=-\mathbf{R}, \mathbf{b}_{2, \mathrm{~S}}=\mathbf{L} / 2 T_{\mathrm{S}}$, and $\mathbf{b}_{3, s}=-\mathbf{L} / 2 T_{\mathrm{S}}$.

It should be noticed that after three samples, i.e. $k-1, k$, and $k+1$, the $k$-th value of the associated state variables can be estimated. The estimation process finishes when all $N$ samples in the interval time are analyzed.

The busbar voltage to be estimated, according to (14), does not require of an initial condition as it is only a function of measurements. Hence, there is no computational effort required to determine the initial steady state.

Once $\mathbf{v}_{r}$ is determined, the receiving end current $\mathbf{i}_{r}$, can be determined as follows,

$$
\mathbf{i}_{r}=\mathbf{i}+\mathbf{i}_{\mathrm{Gr}}+\mathbf{i}_{\mathrm{C} r}
$$

By substituting (9) in (15) yields,

$$
\mathbf{i}_{r}=\mathbf{i}_{s}-\mathbf{i}_{\mathrm{Gs}}-\mathbf{i}_{\mathrm{Cs}}+\mathbf{i}_{\mathrm{Gr}}+\mathbf{i}_{\mathrm{Cr}}
$$

Discretizing and ordering (16) leads to,

$$
\mathbf{i}_{r}^{k}=\mathbf{i}_{s}^{k}-\mathbf{G} \mathbf{v}_{S}^{k}+\mathbf{G} \mathbf{v}_{r}^{k}-\mathbf{C}\left(\mathbf{v}_{S}^{k+1}-\mathbf{v}_{S}^{k-1}\right) / 2 T_{\mathrm{S}}+
$$$$
\mathbf{C}\left(\mathbf{v}_{r}^{k+1}-\mathbf{v}_{r}^{k-1}\right) / 2 T_{\mathrm{S}}
$$

Since $\mathbf{i}_{r}$ is calculated after state variables are estimated (busbar voltages), these variables are named dependent variables.

2) Two busbars connected through a transformer: To analyze the transformer, it is necessary to refer the equivalent circuit either to the primary side of the transformer or to the secondary side. Fig. 1(b) shows the simplified transformer equivalent circuit referred to the primary side. The modeling can be extended for three phase transformer analysis using the appropriate matrix representation of parameters. The transformer model includes the following parameters: the turn ratio $a$ of the ideal transformer; the winding resistance of primary and secondary windings, $\mathbf{R}_{1}$ and $\mathbf{R}_{2}$, respectively; the leakage inductance of primary and secondary windings, $\mathbf{L}_{1}$ and $\mathbf{L}_{2}$, respectively. The state variables are the voltages at the input $\mathbf{v}_{p}$ and at the output $\mathbf{v}_{q}$ of the transformer.

The equation in the continuous-time that represents the three phase transformer is,

$$
\mathbf{v}_{p}-a \mathbf{v}_{q}=\mathbf{R}_{\mathrm{eq}} \mathbf{i}_{\mathrm{T}}+\mathbf{L}_{\mathrm{eq}} \mathbf{i}_{\mathrm{T}}^{\prime}
$$

where $\mathbf{R}_{\text {eq }}=\mathbf{R}_{1}+a^{2} \mathbf{R}_{2}$ and $\mathbf{L}_{\mathbf{e q}}=\mathbf{L}_{1}+a^{2} \mathbf{L}_{2}$.

Solving for $\mathbf{v}_{q}$ in (18) gives,

$$
\mathbf{v}_{q}=a^{-1}\left(\mathbf{v}_{p}-\mathbf{R}_{\mathrm{eq}} \mathbf{i}_{\mathrm{T}}+\mathbf{L}_{\mathrm{eq}} \mathbf{i}_{\mathrm{T}}^{\prime}\right) .
$$

It is assumed that the primary side of the transformer is instrumented. Let $\mathbf{z}_{\mathbf{v}_{\boldsymbol{p}}}$ be the busbar voltages and $\mathbf{z}_{\mathbf{i}_{\mathbf{T}}}$ the transformer current measurements. Hence, (19) is now,

$$
\mathbf{v}_{q}=a^{-1}\left(\mathbf{z}_{\mathbf{v}_{p}}-\mathbf{R}_{\mathrm{eq}} \mathbf{z}_{\mathbf{i}_{\mathrm{T}}}+\mathbf{L}_{\mathrm{eq}} \mathbf{z}_{\mathbf{i}_{\mathrm{T}}}^{\prime}\right) \text {. }
$$

By applying (3) and (4) to discretize (20) yields,

$$
\mathbf{v}_{q}^{k}=a^{-1}\left(\mathbf{z}_{\mathbf{v}_{p}}^{k}-\mathbf{R}_{\mathrm{eq}} \mathbf{z}_{\mathbf{i}_{\mathrm{T}}}^{k}+\mathbf{L}_{\mathrm{eq}}\left(\mathbf{z}_{\mathbf{i}_{\mathrm{T}}}^{k+1}-\mathbf{z}_{\mathbf{i}_{\mathrm{T}}}^{k-1}\right) / 2 T_{\mathrm{S}}\right) .
$$
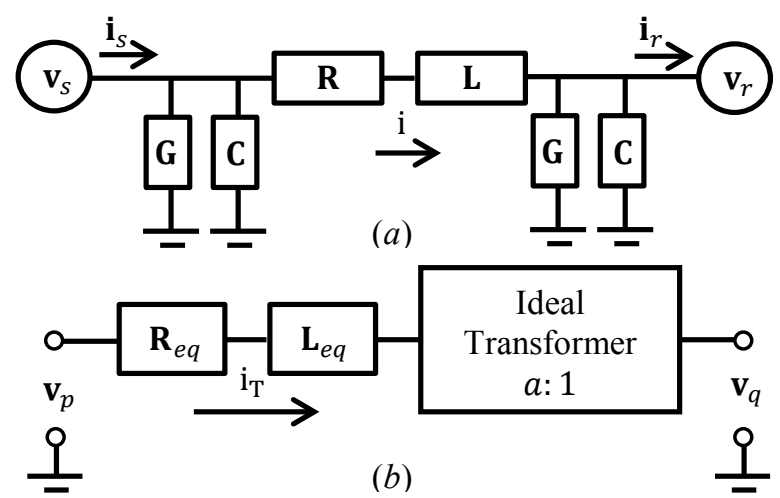

Fig. 1. Circuit models: (a) Tansmission line, $(b)$ Tansformer referred to primary side.

\section{B. Optimal monitoring}

The optimal monitoring system is one that uses the minimal number of measuring devices and their placement so that full observability can be achieved.

1) Number of communication channels: Each instrumented node requires a communication channel. Hence, it is not trivial to place the meter anywhere, e.g. Fig. 2(a) shows two meters placed at node $s$; one for node voltage and one for the sending end line current. This placement generates one communication channel. On the other hand, if the same meters are placed as shown in Fig. 2(b), the number of communication channels is now two as nodes $s$ and $r$ are distant from each other.

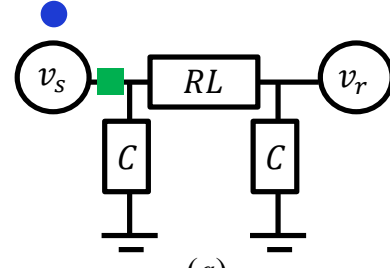

(a)

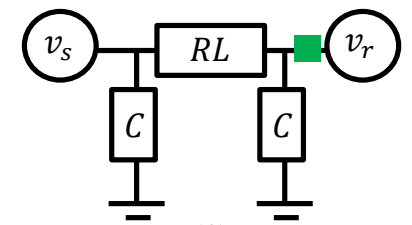

(b)
Fig. 2. Placement of measuring devices: $(a)$ with one communication channel and $(b)$ with two communication channels.

The methods to assess the TSE [10-15] do not use an optimal measurement system. To the authors' knowledge, 
reported methods to assess TSE have not yet proposed a procedure to obtain an optimal placement for the measurement system. However, there are several contributions to obtain full observability in the frequency-domain, i.e. using phasor quantities [25-30]. These contributions can be exploited extending the concept to the time domain.

2) Minimal number of measuring devices: Power system observability analysis can be performed using numerical [25] and topological analysis [26-29]. The topological analysis follows observability rules. The first observability rule establishes that installation of a measuring device in a given node makes itself and other nodes incident to the observable node. The minimum number of instrumented nodes to obtain full observability can be formulated as a problem of Integer Linear Programming (ILP) as follows [26-27],

$$
\operatorname{Min} \sum_{i=1}^{n} \mathbf{d}_{\mathbf{i}}
$$$$
S . T . \text { Ad } \geq \mathbf{b}
$$

$d_{i}=\left\{\begin{array}{lc}1, & \text { if busbar } i \text { is instrumented } \\ 0, & \text { otherwise }\end{array}\right.$

The matrix $\mathbf{A}$ is formed using the line data associated to the node, i.e.

$a_{i j}=\left\{\begin{array}{l}1, \quad \text { if } i=j \\ 1, \quad \text { if } i \text { and } j \text { are adjacents } \\ 0, \quad \text { otherwise }\end{array}\right.$

The vector $\mathbf{d} \in \mathbb{R}^{n}$ contains each possibility of monitoring the $n$ nodes, i.e. $\mathbf{d}=\left[d_{1} d_{2} \cdots d_{n}\right]^{\mathrm{T}}$. The vector $\mathbf{b} \in \mathbb{R}^{n}$ contains the number of times that a busbar is observed. The basic case, when each busbar is observable at least once, corresponds to $\mathbf{b}=\left[\begin{array}{llll}1 & 1 & \cdots & 1\end{array}\right]^{\mathrm{T}}$.

3) Zero injection busbar: There are additional rules that incorporate the concept of zero injection busbar (ZIB). A ZIB is a busbar that does not inject current into the system [28-30]. These rules have been applied to phasor quantities; however, using numerical integration methods, they can be applied in the time-domain. For instance, the series branch current in the transmission line shown in Fig. 1(a) can be determined by,

$$
\mathbf{i}^{k}=\left(\mathbf{R}+\mathbf{L} / T_{\mathrm{S}}\right)^{-1}\left(\mathbf{v}_{\mathrm{S}}^{k}-\mathbf{v}_{r}^{k}+\mathbf{L} \mathbf{i}^{k-1} / T_{\mathrm{S}}\right)
$$

Since $\mathbf{i}^{k-1}$ is not known, it can be initialized to zero and apply the numerical integration for a few cycles to obtain a better initial approximation. The shunt branch can be determined by discretizing (7) and (8), i.e.

$$
\begin{gathered}
\mathbf{i}_{C S}^{k}=\mathbf{C}\left(\mathbf{v}_{s}^{k+1}-\mathbf{v}_{s}^{k-1}\right) / 2 T_{\mathrm{S}} \\
\mathbf{i}_{G S}^{k}=\mathbf{G} \mathbf{v}_{s}^{k}
\end{gathered}
$$

Finally, the sending end current can be determined through the discretized form of (6), i.e.

$$
\mathbf{i}_{s}^{k}=\mathbf{i}^{k}+\mathbf{i}_{C s}^{k}+\mathbf{i}_{G s}^{k}
$$

Similar analysis can be used to determine receiving end currents.

4) Saving index: An analytical way to analyze the effectiveness of the instrumentation used is through a proposed index, named saving index $\eta_{s a}$, defined as follows:

$$
\eta_{s a}=n_{e} /\left(m n_{c h}\right)
$$

where $n_{e}$ is the number of estimated state variables, $m$ the number of measurements, and $n_{c h}$ the number of communication channels. There are two ways to increase $\eta_{s a}$, i.e. by estimating more state variables with the same number of measurements or by estimating the same number of state variables with fewer measurements.

\section{Filtering of noisy measurements during TSE}

Measurements are unfortunately contaminated by noise. In [11], an over-determined solution is used to compensate the adverse effect of noisy measurements. As an alternative, a digital filter is proposed to mitigate the noise of the measurements.

Digital filters can be classified into finite impulse response (FIR) and infinite impulse response (IIR) filters. FIR and IIR filters each have advantages and disadvantages, and none of them is best in all situations. In this research work, an IIR filter is proposed since its design is generally simpler than the one for IIR filter [31-32].

The IIR filter of order $M-1$ can be defined by,

$$
\tilde{z}^{k}=\sum_{i=2}^{M} a_{i} \tilde{z}^{k-i}+\sum_{j=1}^{M} b_{j} z^{k-j}
$$

where $\tilde{\mathrm{Z}}$ is the filtered measurement and $\mathrm{z}$ is the noisy measurement. It is needed to take $M$ samples and set to zero the first $M-1$ outputs to generate the first filtered measurement.

The sample frequency $F_{\mathrm{S}}$ should be considered. According to Nyquist theorem, the $F_{\mathrm{S}}$ must be at least the double of the frequency of interest. Measurements need to be filtered; at cutoff frequency, the attenuation is $-3 \mathrm{~dB}$. Therefore, in order to avoid attenuation at the maximum frequency of interest, the cut-off frequency of the filter is set to ten times over to maximum frequency of interest. Hence,

$$
F_{\mathrm{S}}=20 h f
$$

where $h$ is the maximum harmonic and $f$ is the fundamental frequency.

The techniques to design an IIR filter can be found in the open literature, e.g. [31-32].

\section{Proposed algorithm for TSE}

The proposed algorithm to assess TSE in power systems is shown in the block diagram of Fig. 3. The design of the optimal measuring system and the inverse measuring matrix are previous conditions to run the algorithm. Please notice that the initial state is determined by the first and second samples. The first estimation is performed after the third sample. After state variables are determined, the dependent variables can be computed.

A systematic procedure to identify the fault location is given as follows:

The rms voltage during the pre-disturbance time interval $\left(V_{p r}\right)$ and the rms voltage during the transient time interval $\left(V_{t r}\right)$ of voltages per phase at all nodes can be compared. The place of maximum difference gives a good indication of the fault location. Since there are different voltage areas in a power system, this difference is redefined as follows:

$$
\eta_{t r}=\left(V_{p r}-V_{t r}\right) / V_{p r}
$$




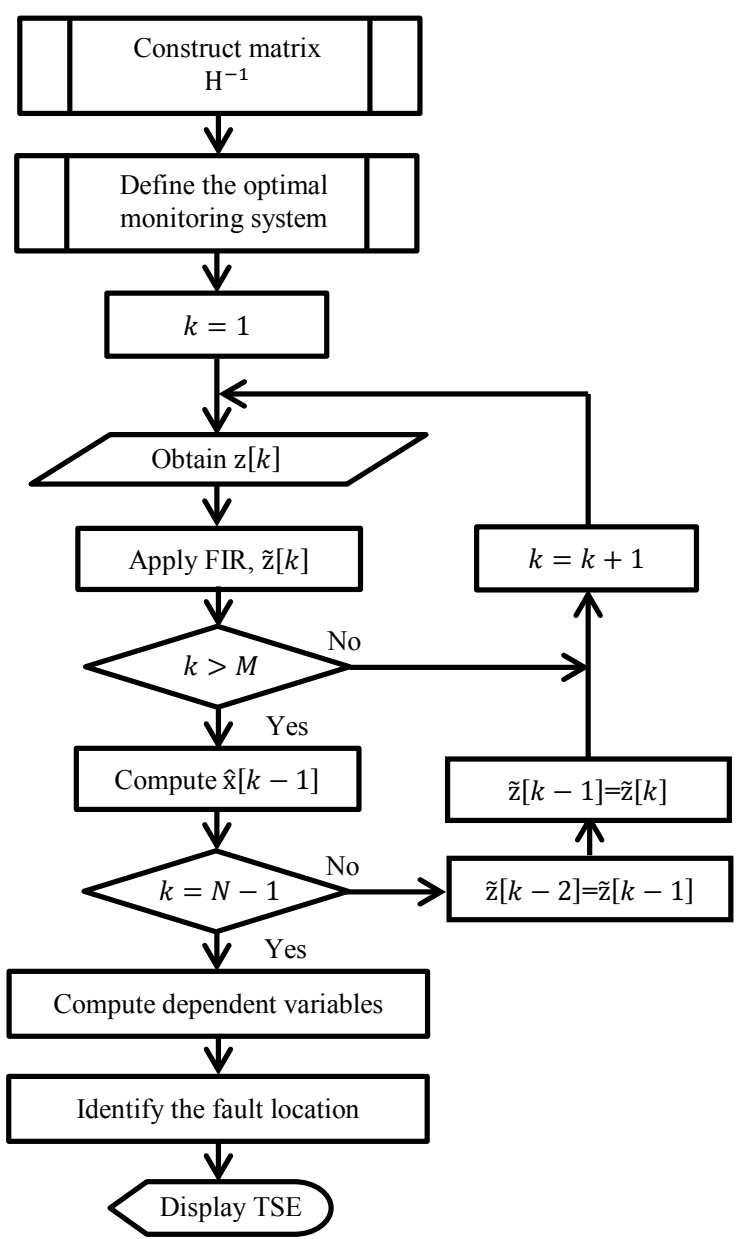

Fig. 3. Flowchart of proposed transient state estimation method.

\section{TEST SySTEMS}

\section{A. Modified IEEE 14-bus test system}

The modified IEEE 14-bus test system is proposed to validate and compare to previous case studies the TSE assessment of a power network. Fig. 4(a) shows the single phase diagram of such test system where the best identified placement of the measuring devices is indicated. The test system parameters are reported in [33] where base values of $100 \mathrm{MVA}$ and $230 \mathrm{KV}$ are assumed. Zero sequence data is given in Table I for unbalanced case studies.

\section{B. Modified New Zealand test system}

The New Zealand $11 \mathrm{kV}$ distribution network is the test system used to validate as well as to compare the proposed methodology to previous case studies for TSE assessment. Fig. 4(b) shows the single line diagram of such test system where the best placement of measuring devices is shown. The positive and zero sequence parameters are reported in [14].

\section{RESULTS}

\section{A. Saving resources}

The main objective of this case study is to evaluate the impact of saving resources in the instrumentation of the power system when an optimal monitoring algorithm is implemented to obtain full observability.

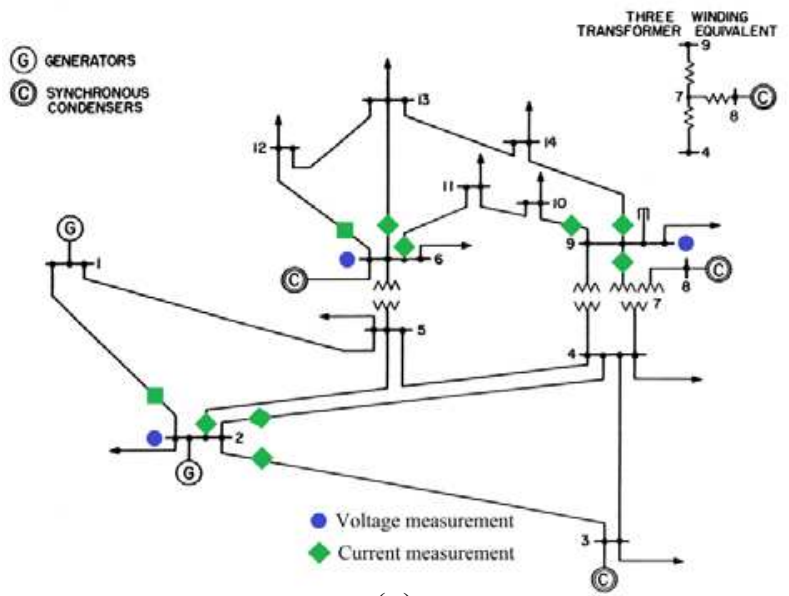

(a)

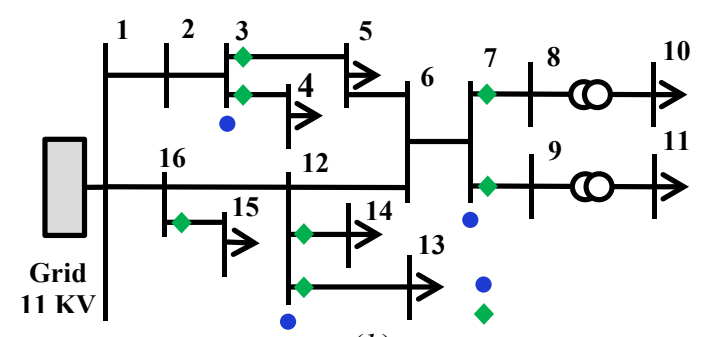

(b)

Fig. 4. Test systems and measuring placement: (a) Modified IEEE 14-bus and (b) New Zealand distribution network.

TABLE I

Zero sequence parameters for modified 14-bus test power system.

\begin{tabular}{|c|c|c|}
\hline Line & Resistance, $\mathbf{R}_{\mathbf{0}}$ & Inductance, $\mathbf{L}_{\mathbf{0}}$ \\
\hline $1-2$ & 0.048450 & 0.147925 \\
\hline $1-5$ & 0.135075 & 0.557600 \\
\hline $2-3$ & 0.117475 & 0.494925 \\
\hline $2-4$ & 0.145275 & 0.440800 \\
\hline $2-5$ & 0.142375 & 0.434700 \\
\hline $3-4$ & 0.167525 & 0.427575 \\
\hline $4-5$ & 0.033375 & 0.105275 \\
\hline $4-7$ & 0 & 0.209120 \\
\hline $4-9$ & 0 & 0.556180 \\
\hline $5-6$ & 0 & 0.252020 \\
\hline $6-11$ & 0.237450 & 0.497250 \\
\hline $6-12$ & 0.307275 & 0.639525 \\
\hline $6-13$ & 0.165375 & 0.325675 \\
\hline $7-8$ & 0 & 0.440375 \\
\hline $7-9$ & 0 & 0.275025 \\
\hline $9-10$ & 0.079525 & 0.211250 \\
\hline $9-14$ & 0.317775 & 0.675950 \\
\hline $10-11$ & 0.205125 & 0.480175 \\
\hline $12-13$ & 0.552300 & 0.499700 \\
\hline $13-14$ & 0.4227325 & 0.870050 \\
\hline
\end{tabular}

1) Measurement set for the modified IEEE 14-bus test system: By applying (22) to the test system shown in Fig. 4(a) and considering the busbar 7 as a ZIB, the resulting instrumented busbars to obtain full observability are 2,6 , and 9 (9 node voltages and 30 nodal currents), as shown in Fig. 4(a). This result agrees with those obtained in [28-30]. To compare the efficiency of the measuring devices placement against the case reported in [11] the saving index defined in (27) is used. The detailed data is given in Table II. Using the proposed methodology the saving index is increased from 0.18 
to 0.35 , i.e. the resources are $94 \%$ more efficient respect to the case reported in [11].

TABLE II

Comparison of required measuring devices

\begin{tabular}{|c|c|c|}
\hline Item & {$[\mathbf{1 1}]$} & Proposed \\
\hline No. of measurements & 33 & 39 \\
\hline No. of communication channels & 5 & 3 \\
\hline Estimated node voltages & 30 & 42 \\
\hline Saving index & 0.18 & 0.35 \\
\hline
\end{tabular}

2) Measurement set for the modified New Zealand distribution test system: By applying (22) to the test system shown of Fig. 4(b) and considering busbars 2, 3, 6, 7, 9, 12, and 15 as ZIBs, the resulting instrumented busbars to obtain full observability are $3,7,12$, and 15 (9 node voltages and 21 nodal line currents) as shown in Fig. 4(b). Data for computing the saving index for this case is given in Table III. The resulting saving index for the proposed methodology has been increased by $81 \%$ with respect to the case reported in [14].

TABLE III

Comparative between proposed and prior placements [14]

\begin{tabular}{|c|c|c|}
\hline Item & {$[\mathbf{1 4}]$} & Proposed \\
\hline No. of measurements & 36 & 30 \\
\hline No. of communication channels & 6 & 4 \\
\hline Estimated node voltages & 48 & 48 \\
\hline Saving index & 0.22 & 0.40 \\
\hline
\end{tabular}

\section{B. Filtering process}

The main objective of case study is to show the performance of the IIR filter implemented to mitigate the noise in measurements. According to (29) and considering $h=50$ and $f=50$, the sampled frequency is $F_{\mathrm{S}}=50,000$ samples per second; thus, the maximum measurable frequency is $25,000 \mathrm{~Hz}$. Then, the cut-off frequency corresponding to the maximum frequency of interest is $2,500 \mathrm{~Hz}$ which is ten times less than $F_{\mathrm{S}} / 2$. The order of filter has been set to $M-1=5$.

The actual values for the test systems shown in Fig. 4 have been taken from simulations using SimPowerSystems toolbox of Simulink ${ }^{\circledR}$. In both test systems, a transient is caused by short-circuit applied in phase A of busbar 5. The measurements are contaminated by adding noise with normal distribution, where the maximum deviation is $5 \%$.

Using (28), the noisy measurements have been filtered. For illustrative purposes, Fig. 5(a) shows the noisy three-phase voltage measurement at busbar 2 in the modified IEEE 14-bus test system of Fig. 4(a); the corresponding filtered measurement is shown in Fig. 5(b). The noise level has been reduced from 5 to $0.5 \%$.

\section{Evaluation of the TSE under an asymmetric fault}

The main objective of this case study is to estimate the TSE at unmonitored busbars and by inspection of the busbars, to identify the cause of the transients.

1) Modified IEEE 14-bus test system: A case of a shortcircuit fault in phase A at busbar 5 (node 13) is evaluated and the results are compared with those obtained with SimPowerSystems tool of Simulink ${ }^{\circledR}$. The short-circuit fault starts at $0.055 \mathrm{~s}$ and ends at $0.12 \mathrm{~s}$. This condition causes a long transient. By using (14) and (21) for the filtered measurements taken according to Fig. 4(a), 13 three-phase busbar voltages can be determined. Using (24-26) and considering busbar 7 as ZIB, the voltage at busbar 8 is estimated. For illustrative purposes, Fig. 6 shows the TSE assessment.

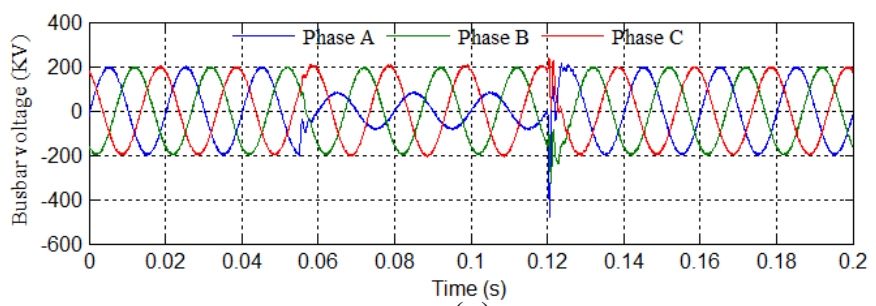

(a)

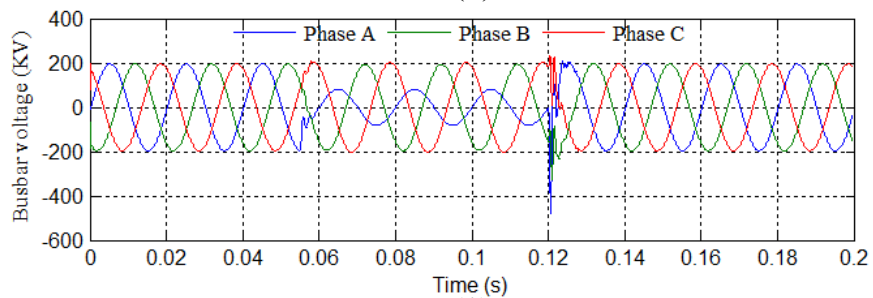

(b)

Fig. 5. Three-phase voltage at busbar 2 in the modified IEEE 14-bus test system. (a) Noisy measurements and $(b)$ filtered measurements.

The TSE assessment for phases A, B, and C, at unmonitored busbar 5 (nodes 13, 14, and 15) are shown in Fig. 6(a), Fig. 6(b) and Fig. 6(c), respectively. It can be observed how variations in phase $A$ affect phases $B$ and $C$ due to magnetic coupling.

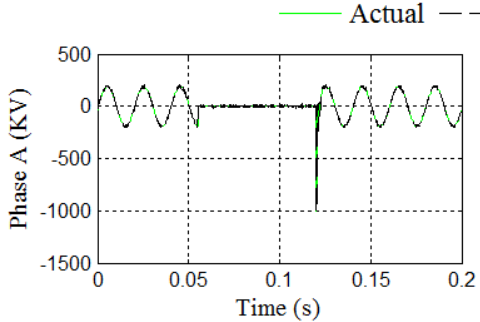

(a)

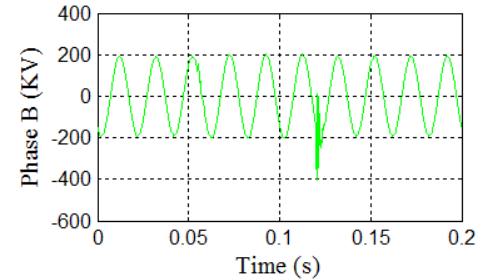

(b)

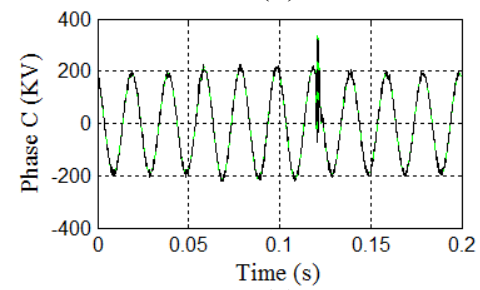

(c)

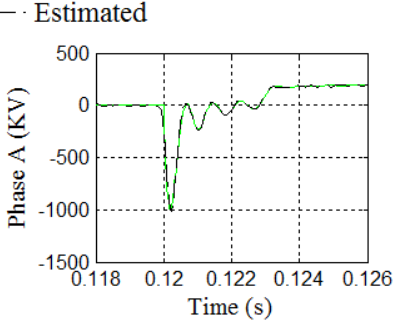

(d)

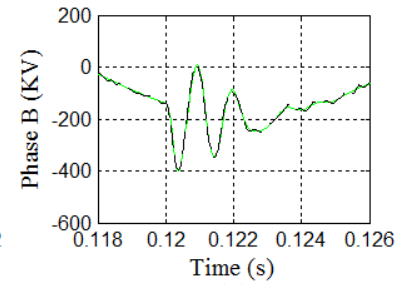

(e)

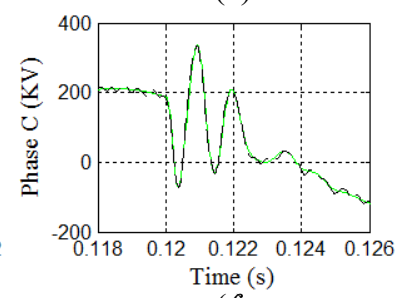

(f)
Fig. 6. Proposed TSE at busbar voltage 5 in the modified IEEE 14-bus test system. The completed time intervals under study are shown in $(a),(b)$, and $(c)$ while, the details of transients are shown in $(d),(e)$ and $(f)$. 
An important issue in transient analysis is to determine the current and voltage maximum variations that can damage some electrical components of the power network as well as the activation of protections. Figs. 6(d), Fig. 6(e), and Fig. 6(f) show that maximum variations occur between 0.118 and 0.126 s. The peak voltage at node 13 is $-1,012 \mathrm{KV}$ with an error of $1.1 \%$, when compared against the actual value.

The main objective of TSE assessment is to identify the fault location. By using (30), the $\eta_{t r}$ for each node voltage is computed. Fig. 7 shows these indices; the maximum $\eta_{t r}$ is 0.9556 , corresponding to phase A of busbar 5 . Thus, the fault location can be estimated, corresponding to node 13 .

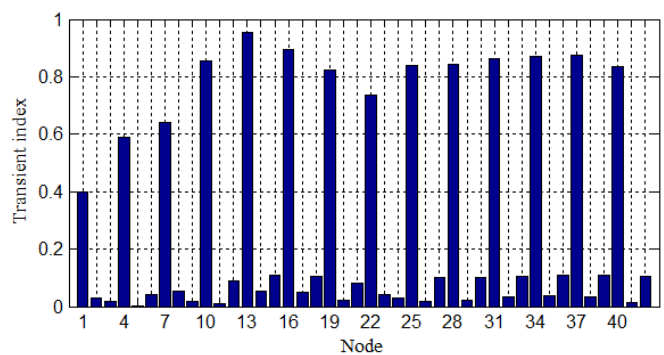

Fig. 7. Fault location in the modified IEEE 14-bus test system using the $\eta_{t r}$.

2) Modified New Zealand test system: The conditions for the modified New Zealand test system are similar to the modified IEEE 14-bus test system. The proposed methodology is applied to estimate the busbar voltages. For illustrative purposes, the estimated busbar voltages at unmonitored busbars 5 (nodes 13, 14, and 15), 11 (nodes 31, 32, and 33), and 16 (nodes 46, 47, and 48) are shown in Fig. 8. Please observe the close agreement between the results obtained with the proposed methodology (estimated) and those obtained by SimPowerSystems toolbox of Simulink ${ }^{\circledR}$ (actual). The maximum error between both responses is approximately $0.58 \%$.

The fault location is estimated using (30). These indices are shown in Fig. 9. The maximum $\eta_{t r}$ is 0.9942 , corresponding to node 13 (phase A of busbar 5). Therefore, it can be suggested that the fault location is at node 13 .

\section{CONCLUSIONS}

A methodology based on numerical derivative methods for transient state estimation has been proposed. It does not require of a precise determination of the pre-disturbance time interval. Thus, allowing a considerable reduction in the computational effort for the TSE assessments.

A method based on time domain topological analysis to obtain the optimal number of monitoring devices for TSE assessment has been proposed. The saving index has been introduced as a criterion to compare the efficiency between two different sets of measuring systems.

The adverse effects of noisy measurement conditions in the TSE assessment have been effectively mitigated using an IIR filter.

The transient index has been introduced as a good tool to estimate the fault location. The tool has been successfully applied in two test systems.
The results obtained with the proposed TSE methodology have been successfully validated through direct comparison against the actual system responses obtained with SimPowerSystems of Simulink ${ }^{\circledR}$. A close agreement between responses has been obtained for all the analyzed cases.
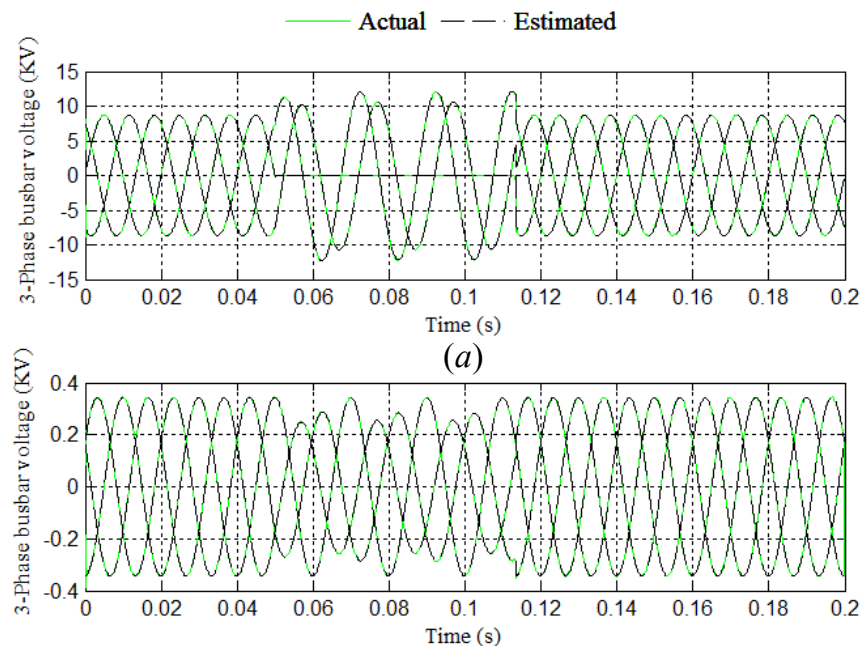

(b)

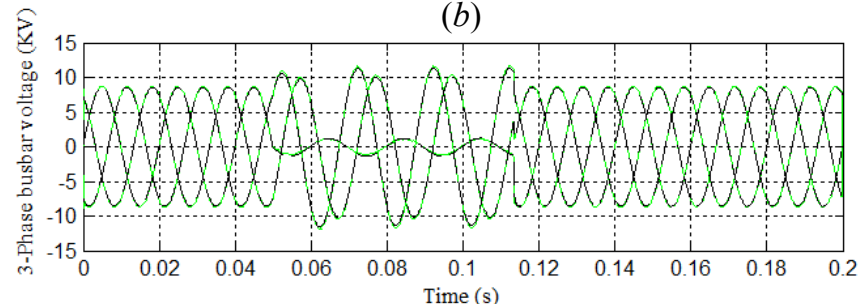

(c)

Fig. 8. Proposed TSE assessment for the modified New Zealand test system at unmonitored busbars $(a) 5,(b) 11$, and $(c) 16$.

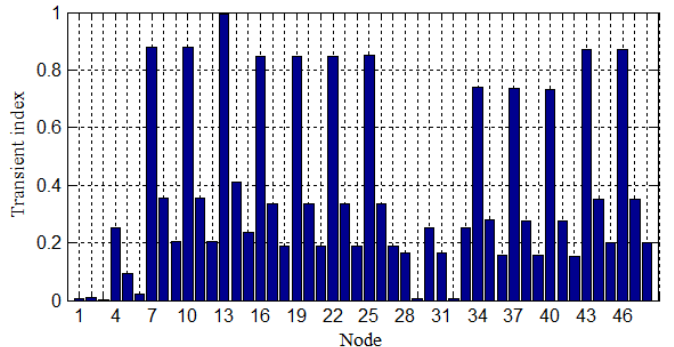

Fig. 9. Fault location in the modified New Zealand test system using the $\eta_{t r}$.

\section{ACKNOWLEDGMENT}

The authors gratefully acknowledge to the Universidad Michoacana de San Nicolás de Hidalgo through the Facultad de Ingeniería Eléctrica, División de Estudios de Posgrado (FIE-DEP) Morelia, México, for the facilities granted to carry out this investigation. The first author wishes to thank the study leave granted by the Instituto Tecnológico de Morelia and to the Consejo Nacional de Ciencia y Tecnología of México (CONACYT) for finance assistance received for his doctoral studies at the FIE-DEP. The second author acknowledges financial support from CONACYT for this investigation.

\section{REFERENCES}

[1] R. C. Dugan, S. Santoso, M. F. McGranaghan, B. H. Wayne, Electrical Power Systems Quality, 3rd ed., McGraw-Hill; 2012. 
[2] J. Arrillaga, N. R. Watson, S. Chen, "Introduction" in Power System Quality Assessment, 2nd ed., New York, NY, John Wiley \& Sons; 2000, pp. $1-17$.

[3] N. R. Watson, "Power Quality State Estimation," Eur. Trans. Elect. Power, vol. 20, pp. 19-33, Jan. 2010.

[4] T. J. Xinyu, H. C. Joe, F. Bruce, M. Deepak, S. George, R. Michael, "Power system state estimation using a direct non-iterative method," Int. J. Elect. Power Energy Syst., vol. 73, pp. 361-368, 2015.

[5] N. S. Da Silva, A. S. Costa, K. A. Clements, E. Andreoli, "Simultaneous estimation of state variables and network topology for power system real-time modelling," Elect. Power Sys. Res., vol. 133, pp. 338-346, 2016.

[6] A. Medina, R. Cisneros-Magaña, "Time-domain harmonic state estimation based on the Kalman filter Poincaré map and extrapolation to the limit cycle," IET Gener. Transm. Distrib., vol. 6, pp. 1209-1217, 2012.

[7] I. Molina-Moreno, A. Medina, R. Cisneros-Magaña, "Time domain harmonic state estimation using filtered measurements based on Fourier transform," in NAPS 2015, Charlotte, NC, Oct 2015, pp. 1-6.

[8] N. C. Woolley, M. Avendaño-Mora, A. P. Woolley, R. Preece, J. V. Milanovi, "Probabilistic estimation of voltage sags using erroneous measurement information," Elect. Power Syst. Res., vol. 90, pp. 142150, 2014.

[9] B. Polajžer, G. Štumberger, D. Dolinar "Detection of voltage sag sources based on the angle and norm changes in the instantaneous current vector written in Clarke's components," Int. J. Elect. Power Energy Syst., vol. 64, pp. 967-976, 2015

[10] K. K. C. Yu, N. R. Watson, "An approximate method for transient state estimation," IEEE Trans. Power Del., vol. 22, no. 3, pp. 1680-1687, Jul. 2007

[11] R. Cisneros-Magaña, A. Medina, "Time domain transient state estimation using singular value decomposition Poincare map and extrapolation to the limit cycle," Int. J. Elect. Power Energy Syst., vol. 53, no. 12, pp. 810-817, Jun. 2013.

[12] A. Farzanehrafat, N. R. Watson, P. Perera, "The Use of transient state estimation for voltage dip/sag assessment," in POWERCON, Auckland, New Zealand, Nov 2012, pp. 1-6.

[13] A. Farzanehrafat, N. R. Watson, "Power quality state estimator for smart distribution grids," IEEE Trans. Power Syst., vol. 28, pp. 2183-2191, 2013.

[14] N. R. Watson, F. Ali, "Three-phase transient state estimation algorithm for distribution systems," IET Gener. Transm. Distrib., vol. 8, no. 10, pp. 1656-1666, 2014.

[15] R. Cisneros-Magaña, A. Medina, J. Segundo-Ramírez, "Efficient time domain power quality state estimation using the enhanced numerical differentiation Newton type method," Int. J. Elect. Power Energy Syst., vol. 63, pp. 414-422, 2014.

[16] N. R. Watson, J. Arrillaga, Power Systems Electromagnetic Transients Simulation. London, U.K.: IET Power and Energy Series 39; 2003.

[17] H. Dommel, "Digital computer solution of electromagnetic transients in single-end multiphase networks," IEEE Trans. Power App. Syst., vol. 88, no. 4, pp.388-399, 1969.

[18] J. Marti, L. Linares, "Real-time EMTP- based transients simulation," IEEE Trans. Power Syst., vol. 9, no. 3, pp. 1309-1317, 1994.

[19] P. Lehn, J. Rittiger, B. Kulicke, "Comparison of the ATP version of the EMTP and the NETOMAC program for simulation of HVDC systems," IEEE Trans. Power Del., vol. 10, no. 4, pp. 2038-2053, 1995.

[20] A. Semlyen, A. Medina, "Computation of the periodic steady state in systems with nonlinear components using a hybrid time and frequency domain methodology," IEEE Trans. Power Syst., vol. 10 no. 3, pp. 1498-1504, 1995.

[21] A. Arefi, M. R. Haghifam, S. H. Fathi, "Observability analysis of electric networks considering branch impedance," Int. J. Electr. Power Energy Syst., vol. 33, no. 4, pp. 954-960, 2011.

[22] I. Rahimi, W. Xu, "A singular value decomposition-based technique for decoupling and analyzing power networks," Int. J. Electr. Power Energy Syst., vol. 74, pp. 265-273, 2016.

[23] R. Burden, J. Fires, Numerical Analysis, 9th ed., CENGAGE Learning; 2011.

[24] J. Grainger, W. Stevenson, Power System Analysis, McGraw-Hill; 1994.

[25] G. Korres, N. Manousakis, T. Xygkis, J. Löfberg, "Optimal phasor measurement unit placement for numerical observability in the presence of conventional measurements using semi definite programming," IET Gener. Transm. Distrib. Vol. 9, no. 15, pp. 2427-2436, 2015.
[26] F. Rashidi, E. Abiri, T. Niknam, M. Reza, “Optimal placement of PMUs with limited number of channels for complete topological observability of power systems under various contingencies," Int. J. Elect. Power Energy Syst., vol. 67, pp. 125-137, 2015.

[27] B. Gou, "Optimal placement of PMUs by integer linear programming," IEEE Trans. Power Syst., vol. 23, no. 3, pp. 1-2, 2008.

[28] H. Mahdi, M. R. Ali, A. Turaj, M. Babak, "Optimal placement of PMUs to maintain network observability using a modified BPSO algorithm," Int. J. Elect. Power Energy Syst., vol. 33, pp. 28-34, 2011.

[29] B. K. Saha, A. K. Sinha, A. K. Pradhan, "An optimal PMU placement technique for power system observability," Int. J. Elect. Power Energy Syst., vol. 42, pp. 71-77, 2012.

[30] M. Arash, S. Heresh, "Optimal PMU placement for power system observability using BICA, considering measurement redundancy," Electr. Power Syst. Res., vol. 103, pp. 78-85, 2013.

[31] T. W. Parks, C. S. Burrus, Digital Filter Design, John Wiley \& Sons; 1987.

[32] V. K. Madisetti, Digital Signal Processing Fundamentals, 2nd ed. CRC Press; 2010.

[33] IEEE 14 bus test system, Power Systems Test Case Archive, https://www.ee.washington.edu/research/pstca/, accessed Oct 24, 2016.

\section{BIOGRAPHIES}

I. Molina-Moreno (M'04) was born in Morelia, México, on May 20, 1964. He graduated from the Instituto Tecnológico de San Luis Potosí, San Luis Potosí, México.

His employment experience includes the Instituto Tecnológico de Morelia. His fields of interest include power quality state estimation.

I. Molina-Moreno received his MSc degree degrees from the Instituto Tecnológico de Morelia, Morelia, México in 2006. He is currently working towards his Ph.D. degree at the Facultad de Ingeniería Eléctrica, UMSNH, Morelia, Mexico.

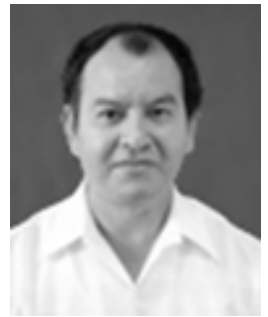

A. Medina (SM'02) obtained his Ph.D. from the University of Canterbury, Christchurch, New Zealand in 1992. He has worked as a Post-Doctoral Fellow at the Universities of Canterbury, New Zealand (1 year) and Toronto, Canada (2 years). He joined the Facultad de Ingeniería Eléctrica, UMSNH, Morelia, México in 1995. He is Senior Member of the IEEE; his research interests are in the analysis of power quality issues, dynamic and steady state analysis of power systems, renewable energy systems and application of advanced numerical and computer techniques to power system analysis.

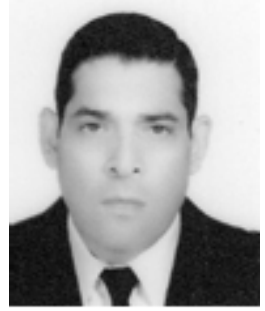

R. Cisneros-Magaña $\left(M^{\prime} 06\right)$ received his $B$. Eng., MSc and Ph.D. degrees in electrical engineering from the Facultad de Ingeniería Eléctrica, UMSNH, Morelia, México in 1982, 2009 and 2013, respectively. His research interests are in modeling and analysis of power systems, power quality, state estimation and renewable energy systems.

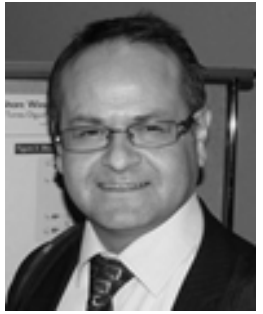

O. Anaya-Lara (M'98) received the B.Eng. and M.Sc. degrees from Instituto Tecnológico de Morelia, Morelia, México, and the Ph.D. degree from the University of Glasgow, Glasgow, U.K., in 1990, 1998, and 2003, respectively. His industrial experience includes periods with Nissan Mexicana, Toluca, México, and CSG Consultants, Coatzacoalcos, México. Currently, he is a Senior Lecturer with the Institute for Energy and Environment, University of Strathclyde, Glasgow, U.K. His current research interests include wind generation, power electronics, and stability of mixed generation power systems. 